\title{
Special Section on Spatial Frequency Domain Imaging
}

Sylvain Gioux

Amaan Mazhar

Anthony J. Durkin

Bruce J. Tromberg

David J. Cuccia 


\section{Special Section on Spatial Frequency Domain Imaging}

\author{
Sylvain Gioux \\ University of Strasbourg \\ iCube Laboratory \\ Strasbourg, France \\ Amaan Mazhar \\ Modulim \\ Irvine, California, United States
}

\section{Anthony J. Durkin}

Beckman Laser Institute and Medical Clinic

University of California, Irvine, United States

\section{Bruce J. Tromberg}

National Institutes of Health

Bethesda, Maryland, United States

David J. Cuccia

Modulim

Irvine, California, United States
This special section of the Journal of Biomedical Optics (JBO) features fourteen articles related to the use of spatial frequency domain imaging (SFDI) as a core methodology for evaluating tissue optical properties. Covering a broad range of topics, this special section presents the latest work in SFDI.

SFDI is a wide-field imaging technique that has garnered significant research attention in the last twenty years. The fundamental principle underlying this technology was first introduced in 1998 by Dögnitz et al., ${ }^{1}$ and quickly matured into a unique way to rapidly image quantitatively large fields-of-view at mesoscopic and macroscopic scales thanks to the developments by Cuccia et al. ${ }^{2,3}$ starting in 2005 . Due to its unique capabilities for quantifying optical properties of living tissues rapidly, the method has gained significant interest from the biomedical optics community with, as of today, more than 130 articles published. In recent years, SFDI has been the topic of dedicated sessions at a number of meetings with contributors from around all the world.

The contributions to this special section of JBO Volume 24, Issue 7 , cover a broad range of topics that best illustrate the activity in this field. There are two review articles covering the use of structured illumination-one at a macroscopic scale, ${ }^{4}$ and a second focused more specifically on SFDI. ${ }^{5}$ A second category of articles focus on modeling in the spatial frequency domain (SFD), including assessment of optical sampling depth in the SFD, ${ }^{6}$ development of analytical models for separation of surface and volumetric scattering, ${ }^{7}$ implantation of machine learning methodology to rapidly extract optical properties, ${ }^{8}$ and derivation of a deterministic radiative transport solver. ${ }^{9} \mathrm{~A}$ third category of articles focus on new instrumentation advances to enable concurrent temporal and spatial modulation of light for rapid oxygenation imaging, ${ }^{10}$ improvement of image quality via a single snapshot of optical

(C) The Authors. Published by SPIE under a Creative Commons Attribution 4.0 Unported License. Distribution or reproduction of this work in whole or in part requires full attribution of the original publication, including its DOI. properties, ${ }^{11}$ and the potential of a single pixel camera in combination with SFDI, ${ }^{12}$ and a hyperspectral SFDI acquisition system. ${ }^{13}$ Finally, there is a group of articles to enhance clinical applications, including characterization of resected cancerous breast tissue ${ }^{14}$ guidance of intra-operative tumor resection, ${ }^{15}$ and correction of light attenuation for improved Cherenkov imaging, ${ }^{16}$ and monitoring of burn wound and skin graft healing. ${ }^{17}$

We thank all the authors and reviewers who contributed to this special section and hope it is a useful resource. We also thank the JBO staff for their assistance and support. We hope that the work featured in this special section will provide a launchpad for others to use SFDI for fundamental research as well as practical clinical applications.

\section{References}

1. N. Dögnitz and G. Wagnieres, "Determination of tissue optical properties by steady-state spatial frequency-domain reflectometry," Lasers Med. Sci. 13(1), 55-65 (1998).

2. D. J. Cuccia et al., "Modulated imaging: quantitative analysis and tomography of turbid media in the spatial-frequency domain," Opt. Lett. 30(11), 1354-1356 (2005).

3. D. J. Cuccia et al., "Quantitation and mapping of tissue optical properties using modulated imaging," J. Biomed. Opt. 14(2), 024012 (2009)

4. J. P. Angelo et al., "Review of structured light in diffuse optical imaging," J. Biomed. Opt. 24(7), 071602 (2018).

5. S. A. Gioux, A. Mazhar, and D. J. Cuccia, "Spatial frequency domain imaging in 2019: principles, applications, and perspectives," J. Biomed. Opt. 24(7), 071613 (2019).

6. C. K. Hayakawa et al., "Optical sampling depth in the spatial frequency domain," J. Biomed. Opt. 24(7), 071603 (2018).

7. S. Nothelfer et al., "Spatial frequency domain imaging using an analytical model for separation of surface and volume scattering," J. Biomed. Opt. 24(7), 071614 (2018).

8. S. Panigrahi and S. Gioux, "Machine learning approach for rapid and accurate estimation of optical properties using spatial frequency domain imaging," J. Biomed. Opt. 24(7), 071606 (2018). 
9. S. T. Horan et al., "Recovery of layered tissue optical properties from spatial frequency-domain spectroscopy and a deterministic radiative transport solver," J. Biomed. Opt. 24(7), 071607 (2018).

10. M. Schmidt et al., "Real-time, wide-field, and quantitative oxygenation imaging using spatiotemporal modulation of light," J. Biomed. Opt. 24(7), 071610 (2019).

11. E. Aguénounon et al., "Single snapshot of optical properties image quality improvement using anisotropic two-dimensional windows filtering," J. Biomed. Opt. 24(7), 071611 (2019).

12. E. Aguénounon et al., "Single snapshot imaging of optical properties using a single-pixel camera: a simulation study," J. Biomed. Opt. 24(7), 071612 (2019).

13. M. Torabzadeh et al., "Hyperspectral imaging in the spatial frequency domain with a supercontinuum source," J. Biomed. Opt. 24(7), 071614 (2019).
14. D. M. McClatchy III et al., "Light scattering measured with spatial frequency domain imaging can predict stromal versus epithelial proportions in surgically resected breast tissue," J. Biomed. Opt. 24(7), 071605 (2018).

15. D. Wirth et al., "Feasibility of using spatial frequency-domain imaging intraoperatively during tumor resection," J. Biomed. Opt. 24(7), 071608 (2018).

16. R. Hachadorian et al., "Correcting Cherenkov light attenuation in tissue using spatial frequency domain imaging for quantitative surface dosimetry during whole breast radiation therapy," J. Biomed. Opt. 24(7), 071609 (2018).

17. G. Kennedy et al., "Spatial frequency domain imaging; a quantitative, non-invasive tool for in-vivo monitoring of burn wound and skin graft healing," J. Biomed. Opt. 24(7), 071615 (2019). 